\title{
Desenvolvimento inicial de Pyrrhulina australis Eigenmann \& Kennedy, 1903 (Characiformes, Lebiasinidae)
}

\author{
Tatia Leika Taguti ${ }^{1,2}$, Darlon Kipper ${ }^{1,2}$, Andréa Bialetzki ${ }^{1,2,5}$, Paulo Vanderlei Sanches ${ }^{3,4}$, \\ Maristela Cavicchioli Makrakis ${ }^{3}$, Gilmar Baumgartner ${ }^{3}$ \& Rodrigo Fernandes $^{2}$ \\ ${ }^{1}$ Laboratório de Ictioplâncton, Núcleo de Pesquisas em Limnologia, Ictiologia e Aqüicultura - Nupélia, \\ Universidade Estadual de Maringá - UEM, \\ Av. Colombo, 5790, bloco G-90, CEP 87020-900, Maringá, PR, Brasil, e-mail: bialetzki@nupelia.uem.br \\ ${ }^{2}$ Pós-Graduação em Ecologia de Ambientes Aquáticos Continentais - PEA, \\ Departamento de Biologia - DBI, Universidade Estadual de Maringá - UEM, Maringá, PR, Brasil \\ ${ }^{3}$ Universidade Estadual do Oeste do Paraná - UNIOESTE, \\ Rua da Faculdade, 645, Jd. La Salle, CEP 85903-000, Toledo, PR, Brasil \\ ${ }^{4}$ Universidade Paranaense - UNIPAR, \\ Av. Parigot de Souza, 3636, CEP 85903-170, Toledo, PR, Brasil \\ ${ }^{5}$ Autor para correspondência: Andréa Bialetzki,e-mail: bialetzki@nupelia.uem.br
}

TAGUTI, T.L., KIPPER, D., BIALETZKI, A., SANCHES, P.V., MAKRAKIS, M.C., BAUMGARTNER, G. \& FERNANDES, R. Early development of Pyrrhulina australis Eigenmann \& Kennedy, 1903 (Characiformes, Lebiasinidae). Biota Neotrop. 9(4): http://www.biotaneotropica.org.br/v9n4/en/ abstract?article+bn01709042009.

\begin{abstract}
This work describes the initial development of Pyrrhulina australis Eigenmann \& Kennedy, 1903, the only known Lebiasinidae of the upper Paraná River floodplain. Eighty specimens were analyzed, sixty larvae and twenty juveniles, collected between 1992 and 2005 in several areas (rivers, channels and ponds). From each specimen morphometric and meristic data were obtained and, subsequently, growth patterns were analyzed. Larvae presented greater pigment concentrations in the superior region of the intestine, head and around the mouth. Notochord is characterized by possessing a protrusion that later becomes part of the caudal fin. The fin formation sequence is: caudal, dorsal, anal, pelvic and pectoral. In relation to myomeres we observed that their numbers ranged from 23 to 32 (12-20 preanal and 10-16 postanal). Growth pattern analyses revealed that nose length and eye diameter were allometric, whereas head height and length, as well as body height and pre-pectoral distance were better represented by the piecewise model, revealing that most of the body metamorphosis occurs during the transitional breakpoint between the larval and juvenile periods.
\end{abstract}

Keywords: Charutinho, ontogeny, larvae, juvenile, Paraná River.

TAGUTI, T.L., KIPPER, D., BIALETZKI, A., SANCHES, P.V., MAKRAKIS, M.C., BAUMGARTNER, G. \& FERNANDES, R. Desenvolvimento inicial de Pyrrhulina australis Eigenmann \& Kennedy, 1903 (Characiformes, Lebiasinidae). Biota Neotrop. 9(4): http://www.biotaneotropica. org.br/v9n4/pt/abstract?article+bn01709042009.

Resumo:Este trabalho descreve o desenvolvimento inicial de Pyrrhulina australis Eigenmann \& Kennedy, 1903, o único Lebiasinidae da planície alagável do Alto Rio Paraná. Para isso, foram analisados 80 indivíduos, sendo 60 larvas e 20 juvenis, coletados entre os anos de 1992 e 2005, em vários ambientes (rios, canais e lagoas). De cada exemplar, foram obtidos dados morfométricos e merísticos e, posteriormente, o padrão de crescimento entre as variáveis morfométricas foi analisado. As larvas apresentaram grande concentração de pigmentos na região superior do intestino, da cabeça e ao redor da boca. A notocorda foi caracterizada por possuir um prolongamento que, posteriormente, diferenciou-se em um raio da nadadeira caudal. A sequiência de formação das nadadeiras foi: caudal, dorsal, anal, pélvica e peitoral. Em relação aos miômeros, observou-se que estes variaram de 23 a 32 (12-20 pré- e 10-16 pós-anal). A análise do crescimento revelou que o comprimento do focinho e o diâmetro do olho foram alométricos, enquanto a altura e o comprimento da cabeça, bem como a altura do corpo e a distância pré-peitoral apresentaram crescimento abrupto, evidenciando diferentes taxas de crescimento entre os períodos larval e juvenil. Esses resultados revelam que neste intervalo ocorre grande parte da metamorfose na espécie.

Palavras-chave: Charutinho, ontogenia, larvas, juvenis, Rio Paraná. 


\section{Introdução}

A região Neotropical engloba a América do Sul, que possui a maior fauna de peixes de água doce conhecida, sendo que só no Brasil são encontradas mais de 2.500 espécies válidas (Graça \& Pavanelli 2007). No entanto, apesar desse grande número, ainda pouco se conhece sobre o desenvolvimento inicial das espécies.

O estudo de ovos e larvas de peixes tem grande importância para o entendimento dos processos reprodutivos e das formas de relacionamento da comunidade íctica com o ambiente. Entretanto, um dos maiores obstáculos encontrados nesta área é a dificuldade de identificar o material coletado em ambiente natural (Bialetzki et al. 1998), uma vez que existe grande similaridade morfológica entre as espécies nos primeiros estágios de desenvolvimento (Snyder 1981, Fuiman et al. 1983). Soma-se a isso, a grande escassez de bibliografia que permite uma correta identificação dos peixes nesta fase de desenvolvimento.

Lebiasinidae é uma família de peixes de água doce encontrada na Costa Rica, Panamá e América do Sul (Weitzman, M\& Weitzman, S.H. 2003). São conhecidos pelos aquariofilistas como "peixes-lápis", devido às listras coloridas do corpo e à postura com a cabeça continuamente apontando para cima (Buckup 1999). Geralmente apresentam pequeno porte, possuem o corpo cilíndrico, linha lateral reduzida ou ausente e a nadadeira adiposa presente ou não (Weitzman, M \& Weitzman, S.H. 2003). Na região do Alto Rio Paraná, apenas Pyrrhulina australis Eigenmann \& Kennedy, 1903 é registrada (Graça \& Pavanelli 2007).

Aspectos do desenvolvimento inicial de Lebiasinidae são restritos aos trabalhos de Ponton \& Mérigoux (2001), que descreveram as larvas de Copella carsevennensis (Regan, 1912) e Pyrrhulina filamentosa Valenciennes, 1847, e de Nakatani et al. (2001), que descreveram sucintamente o desenvolvimento de P. australis. Assim, com o objetivo de contribuir para o conhecimento do desenvolvimento inicial dos peixes neotropicais, este estudo apresenta detalhadamente a descrição de larvas e juvenis de $P$. australis coletados no ambiente natural, baseada em aspectos morfométricos, merísticos e na pigmentação. As alterações destas características ao longo do desenvolvimento ontogênico também são analisadas.

\section{Material e Métodos}

Devido às dificuldades inerentes ao estudo de ovos e larvas, os indivíduos utilizados neste estudo são provenientes de um grande esforço amostral, envolvendo um longo período de amostragem entre 1992 e 2005, em 42 estações de coleta distribuídas em diversos ambientes (rios, canais e lagoas) da planície alagável do Alto Rio Paraná (Figura 1).

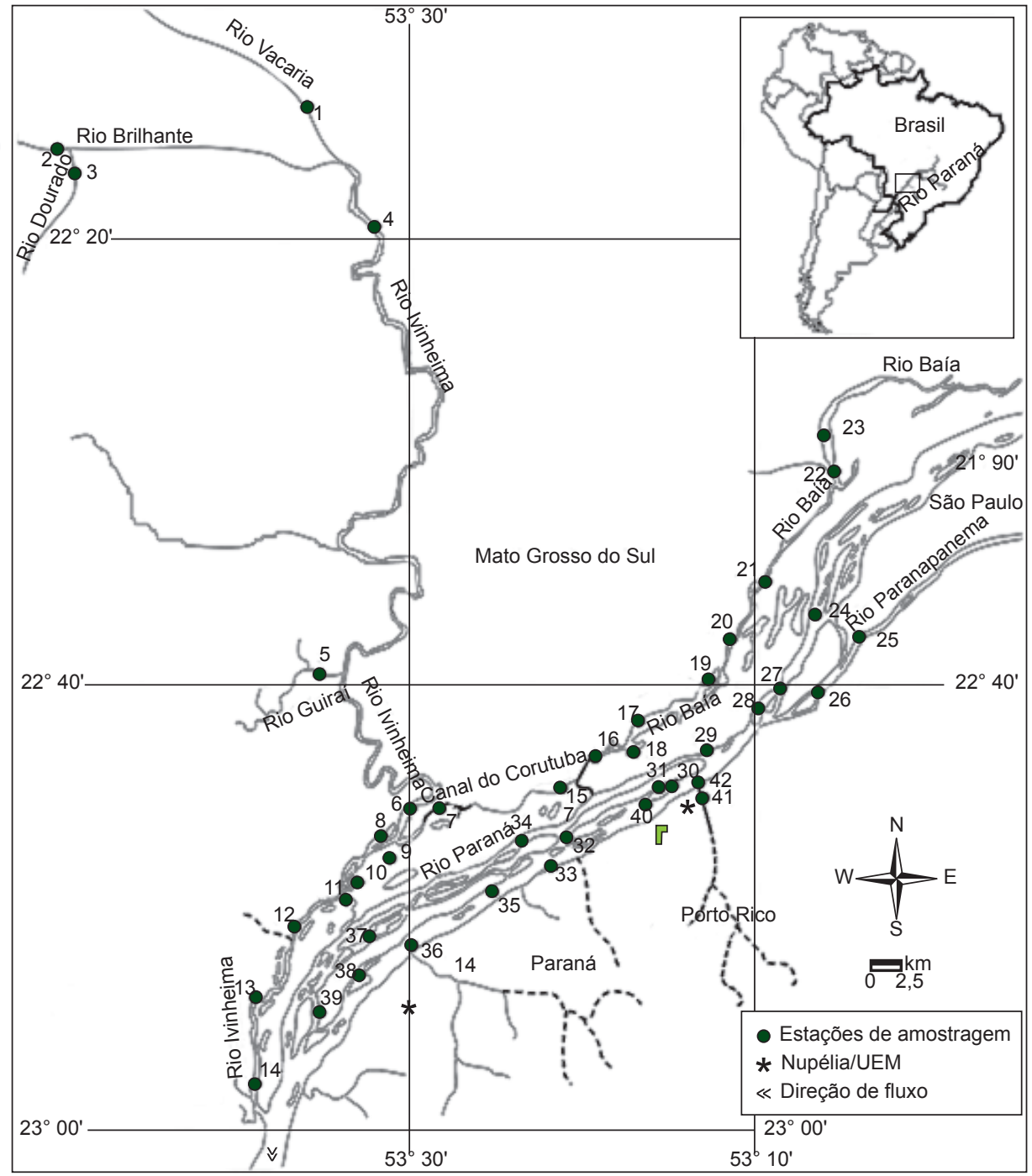

Figure 1. Location of the sampling sites in the upper Paraná River floodplain, Brazil.

Figura 1. Localização das estações amostradas na planície alagável do Alto Rio Paraná, Brasil. 
Para a captura, foram realizadas coletas na região limnética, utilizando-se redes de plâncton cônico-cilíndricas, e na região litorânea, utilizando-se peneirão e redes de arrasto, conforme descrito por Nakatani et al. (2001). O material obtido foi fixado em formol diluído a 4\%, tamponado com carbonato de cálcio.

Em laboratório, as larvas e juvenis capturados foram separados do restante do plâncton, com auxílio de estereomicroscópio, e, em seguida, identificados através da técnica de seqüência regressiva de desenvolvimento (Ahlstrom \& Moser 1976), que consiste na comparação morfológica de indivíduos menores a partir da forma juvenil conhecida. Em seguida, os indivíduos foram enquadrados nos períodos larval e juvenil (caracterizado pela completa formação dos raios das nadadeiras e surgimento de escamas), sendo as larvas classificadas em quatro estágios (larval vitelino, pré-flexão, flexão e pós-flexão), de acordo com o grau de flexão da notocorda e desenvolvimento da nadadeira caudal e seus elementos de suporte, segundo Ahlstrom \& Ball (1954), modificado por Nakatani et al. (2001). A descrição de cada período foi baseada no grau de desenvolvimento e na ocorrência dos principais eventos morfológicos, sendo ilustrados, com auxílio de câmara clara, indivíduos que melhor representassem estas características. As larvas e juvenis utilizados na fundamentação da pesquisa encontram-se depositados na coleção do Laboratório de Ictioplâncton do Núcleo de Pesquisas em Limnologia, Ictiologia e Aqüicultura (Nupélia) da Universidade Estadual de Maringá - PR.

Para a caracterização do desenvolvimento inicial, foram tomadas, através de estereomicroscópio equipado com retículo micrométrico, as seguintes medidas corporais expressas em milímetros (Ahlstrom et al. 1976): comprimento padrão (CP), comprimento da cabeça (CC), comprimento do focinho (CF), diâmetro do olho (DOL), altura da cabeça (AC), altura do corpo (ACO) e distâncias pré-peitoral (FNP), pré-pélvica (FNV), pré-dorsal (FND) e pré-anal (FNA). Para análise das relações corporais (expressas como porcentagem) ao longo do desenvolvimento, as variáveis CC, ACO, FNP, FNL, FND e FNA foram relacionadas com o comprimento padrão, enquanto $\mathrm{CF}$, DOL e $\mathrm{AC}$ foram relacionadas ao comprimento da cabeça. As relações corporais para a altura do corpo $(\mathrm{ACO} / \mathrm{CP})$, comprimento da cabeça $(\mathrm{CC} / \mathrm{CP})$ e diâmetro do olho (DOL/CC) foram estabelecidas utilizando-se os critérios sugeridos por Leis \& Trnski (1989). Para a caracterização merística foram contados, quando possível, o número de miômeros totais, pré e pós-anal, e os raios das nadadeiras peitoral (P), pélvica (V), dorsal (D) e anal (A).

Para examinar o padrão de crescimento da espécie, as variáveis morfométricas (variáveis dependentes) foram plotadas contra os comprimentos padrão e da cabeça (variáveis independentes) e suas relações foram descritas por diferentes modelos de crescimento, os quais podem representar diferentes processos biológicos (Kováč et al. 1999). Inicialmente, a hipótese de que o desenvolvimento das relações corporais é isométrico foi testada a partir de uma regressão linear simples. Conjuntamente à hipótese de isometria, foram testadas as hipóteses alternativas de desenvolvimento gradualmente alométrico (regressão quadrátrica) ou abrupto (regressão linear do tipo piecewise), o qual é marcado por pontos de quebra que refletem diferentes taxas de crescimento. A seleção do melhor modelo de crescimento para cada variável morfométrica em relação ao tamanho do corpo foi testada através de testes F (Sokal \& Rohlf 1981). O nível de significância adotado para as análises foi $\mathrm{p}<0,05$.

\section{Resultados}

Foram analisados 80 indivíduos, sendo 20 em estágio de préflexão, 20 em flexão, 20 em pós-flexão e 20 juvenis. Indivíduos no estágio de larval vitelino não foram incluídos por não terem sido capturados. A descrição de cada período é apresentada a seguir e ilustrada na Figura 2a-f. Os resultados referentes às variáveis morfométricas e merísticas são apresentados na Tabela 1 .

\section{Caracterização do período larval}

Estágio de pré-flexão (Figura 2a): O comprimento padrão varia de 3,00 a 4,45 mm. O saco vitelino está presente, entretanto no final deste estágio encontra-se quase absorvido. A pigmentação é constituída por cromatóforos puntiformes, concentrados na superfície dorsal da
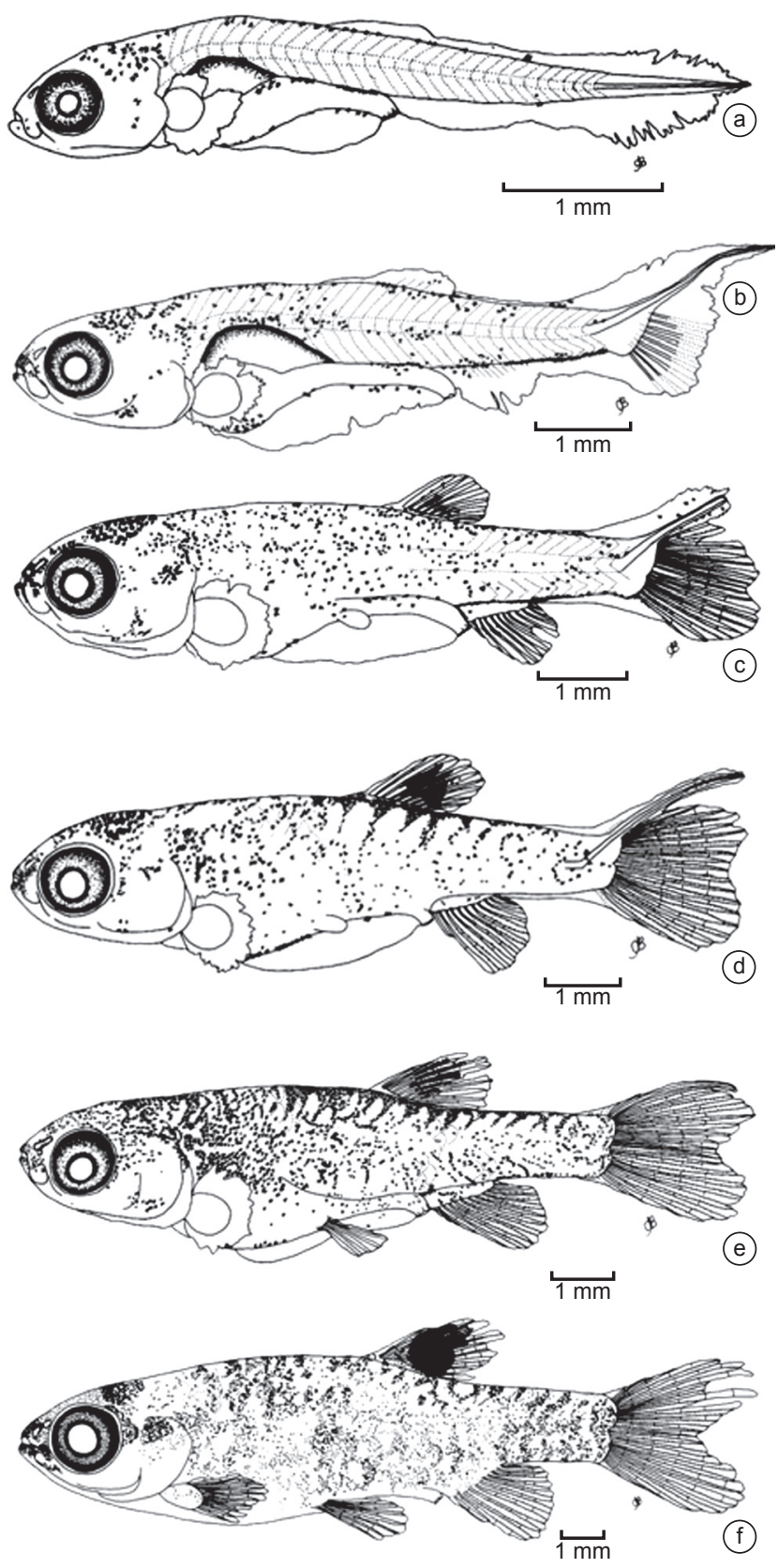

Figure 2. Early development of Pyrrhulina australis. a) Preflexion (4.62 mm $\mathrm{CP})$; b) Flexion (6.29 mm CP); c) Early postflexion (7.20 mm CP); d) Intermediate postflexion (7.93 $\mathrm{mm} \mathrm{CP})$; e) Late postflexion $(8.90 \mathrm{~mm} \mathrm{CP})$ and f) Juvenile (14.00 $\mathrm{mm} \mathrm{CP})($ Scale $=1 \mathrm{~mm})$.

Figura 2. Desenvolvimento inicial de Pyrrhulina australis. a) Pré-flexão (4,62 mm CP); b) Flexão (6,29 mm CP); c) início de Pós-flexão (7,20 mm CP); d) Pós-flexão intermediário $(7,93 \mathrm{~mm} \mathrm{CP})$; e) final de Pós-flexão $(8,90 \mathrm{~mm}$ CP) e f) Juvenil $(14,00 \mathrm{~mm} \mathrm{CP})($ Escala $=1 \mathrm{~mm})$. 
Table 1. Minimum (Min) and maximum (Max) values, mean $(\overline{\mathrm{X}})$ and standard deviation (DP) (mm and \%) of the morphometric and meristic variables, obtained in larvae and juveniles of Pyrrhulina australis ( $\mathrm{PF}=$ preflexion; $\mathrm{FL}=$ flexion; $\mathrm{FP}=$ postflexion; $\mathrm{J}=$ juvenile; $\mathrm{NV}=$ not visible).

Tabela 1. Valores mínimos (Min), máximos (Max), médias ( $\bar{X})$ e desvio padrão (DP) (mm e \%) para as variáveis morfométricas e merísticas, obtidos de larvas e juvenis de Pyrrhulina australis ( $\mathrm{PF}=$ pré-flexão; $\mathrm{FL}=$ flexão; $\mathrm{FP}$ = pós-flexão; $\mathrm{J}=$ juvenis; $\mathrm{NV}$ = não visível).

\begin{tabular}{|c|c|c|c|c|c|c|c|c|}
\hline \multirow{3}{*}{$\begin{array}{l}\text { Variáveis } \\
(\mathbf{m m})\end{array}$} & \multicolumn{6}{|c|}{ Período larval } & \multirow{2}{*}{\multicolumn{2}{|c|}{$\begin{array}{c}\text { Período juvenil } \\
\mathbf{J}(\mathbf{n}=\mathbf{2 0}) \\
\end{array}$}} \\
\hline & \multicolumn{2}{|c|}{$P F(n=20)$} & \multicolumn{2}{|c|}{ FL $(n=20)$} & \multicolumn{2}{|c|}{$F P(n=20)$} & & \\
\hline & Min/Max & $\overline{\mathbf{X}} \pm \mathbf{D P}$ & Min/Max & $\overline{\mathbf{X}} \pm \mathbf{D P}$ & Min/Max & $\overline{\mathbf{X}} \pm \mathbf{D P}$ & Min/Max & $\overline{\mathbf{X}} \pm \mathbf{D P}$ \\
\hline $\mathrm{CP}$ & $3,00-4,45$ & $3,61 \pm 0,42$ & $5,33-7,33$ & $6,03 \pm 0,54$ & $7,40-13,28$ & $9,66 \pm 2,29$ & $13,14-31,00$ & $19,67 \pm 5,67$ \\
\hline $\mathrm{AC}$ & $0,40-0,70$ & $0,51 \pm 0,07$ & $0,73-1,40$ & $1,01 \pm 0,19$ & $1,40-2,86$ & $2,00 \pm 0,51$ & $2,71-5,00$ & $3,75 \pm 0,73$ \\
\hline $\mathrm{CC}$ & $0,50-0,95$ & $0,68 \pm 0,14$ & $1,07-1,90$ & $1,44 \pm 0,27$ & $2,00-4,00$ & $2,83 \pm 0,66$ & $3,57-7,57$ & $5,23 \pm 1,20$ \\
\hline $\mathrm{CF}$ & $0,05-0,20$ & $0,13 \pm 0,04$ & $0,20-0,40$ & $0,28 \pm 0,06$ & $0,37-0,86$ & $0,55 \pm 0,16$ & $0,71-2,14$ & $1,24 \pm 0,42$ \\
\hline DOL & $0,20-0,40$ & $0,30 \pm 0,05$ & $0,40-0,80$ & $0,58 \pm 0,12$ & $0,77-1,60$ & $1,11 \pm 0,28$ & $1,43-2,71$ & $1,91 \pm 0,38$ \\
\hline $\mathrm{ACO}$ & $0,45-0,75$ & $0,52 \pm 0,07$ & $0,67-1,70$ & $1,16 \pm 0,34$ & $1,90-3,43$ & $2,47 \pm 0,60$ & $3,28-8,29$ & $4,90 \pm 1,33$ \\
\hline FNP & $0,50-1,00$ & $0,69 \pm 0,14$ & $1,17-2,00$ & $1,53 \pm 0,26$ & $2,20-4,00$ & $2,97 \pm 0,65$ & $4,00-7,86$ & $5,26 \pm 1,21$ \\
\hline FNV & NV & $\mathrm{NV}$ & NV & $\mathrm{NV}$ & $3,80-7,28$ & $5,05 \pm 1,29$ & $6,71-15,50$ & $9,97 \pm 2,58$ \\
\hline FND & NV & NV & $\mathrm{NV}$ & $\mathrm{NV}$ & $4,30-8,43$ & $5,82 \pm 1,46$ & $7,86-18,10$ & $12,05 \pm 3,37$ \\
\hline FNA & $\mathrm{NV}$ & NV & $\mathrm{NV}$ & $\mathrm{NV}$ & $5,20-9,71$ & $6,88 \pm 1,77$ & $9,28-22,30$ & $14,47 \pm 4,33$ \\
\hline \multicolumn{9}{|l|}{$\begin{array}{c}\text { Relações } \\
(\%)\end{array}$} \\
\hline $\mathrm{AC} / \mathrm{CC}$ & $60-100$ & $76,22 \pm 12,91$ & $53,33-82,30$ & $70,31 \pm 6,7$ & $61,98-78,99$ & $70,55 \pm 4,16$ & $64,58-77,72$ & $72,14 \pm 3,99$ \\
\hline $\mathrm{CF} / \mathrm{CC}$ & $10-25$ & $19,21 \pm 5,54$ & $13,33-26,67$ & $19,83 \pm 3,37$ & $15,55-27,56$ & $19,38 \pm 2,50$ & $16,59-28,27$ & $23,2 \pm 3,22$ \\
\hline DOL/CC & $35,71-58,33$ & $44,67 \pm 6,73$ & $31,33-46,90$ & $39,96 \pm 3,72$ & $29,28-45,83$ & $39,23 \pm 3,18$ & $23,95-42,15$ & $37,01 \pm 4,01$ \\
\hline $\mathrm{CC} / \mathrm{CP}$ & $14,29-22,35$ & $18,79 \pm 2,31$ & $19,56-28,78$ & $23,86 \pm 3,11$ & $25,74-32,82$ & $29,37 \pm 1,99$ & $22,63-31,06$ & $27,06 \pm 2,23$ \\
\hline $\mathrm{ACO} / \mathrm{CP}$ & $12,50-17,65$ & $14,41 \pm 1,12$ & $12,57-27,30$ & $18,98 \pm 4,33$ & $23,56-27,48$ & $25,57 \pm 1,18$ & $21,55-30,93$ & $25,15 \pm 2,16$ \\
\hline FNP/CP & $12,20-22,47$ & $19,13 \pm 2,93$ & $21,94-31,58$ & $25,22 \pm 2,9$ & $27,94-34,35$ & $30,84 \pm 1,82$ & $17,11-30,75$ & $27,34 \pm 3,13$ \\
\hline $\mathrm{FNV} / \mathrm{CP}$ & NV & $\mathrm{NV}$ & NV & $\mathrm{NV}$ & $50,00-54,82$ & $52,07 \pm 1,41$ & $45,25-56,29$ & $51,13 \pm 2,65$ \\
\hline $\mathrm{FND} / \mathrm{CP}$ & NV & NV & NV & NV & $56,58-63,48$ & $60,12 \pm 1,79$ & $50,83-64,22$ & $60,37 \pm 3,97$ \\
\hline FNA/CP & $\mathrm{NV}$ & NV & $\mathrm{NV}$ & $\mathrm{NV}$ & $69,17-74,42$ & $70,97 \pm 1,79$ & $64,17-75,73$ & $72,10 \pm 2,45$ \\
\hline \multicolumn{9}{|l|}{ Miômeros } \\
\hline Total & $23-32$ & $28 \pm 2,09$ & $29-32$ & $30 \pm 1,12$ & $\mathrm{NV}$ & NV & $\mathrm{NV}$ & $\mathrm{NV}$ \\
\hline Pré-anal & $12-17$ & $15 \pm 1,27$ & $16-20$ & $17 \pm 1,16$ & NV & NV & NV & NV \\
\hline Pós-anal & $10-15$ & $13 \pm 1,24$ & $11-14$ & $13 \pm 1,29$ & $\mathrm{NV}$ & $\mathrm{NV}$ & $\mathrm{NV}$ & $\mathrm{NV}$ \\
\hline \multicolumn{9}{|l|}{ Raios } \\
\hline $\mathrm{P}$ & $\mathrm{NV}$ & NV & $\mathrm{NV}$ & $\mathrm{NV}$ & $\mathrm{NV}$ & $\mathrm{NV}$ & $8-14$ & $11 \pm 1,28$ \\
\hline V & NV & NV & $\mathrm{NV}$ & $\mathrm{NV}$ & 7-9 & $8 \pm 0,58$ & $7-9$ & $8 \pm 0,52$ \\
\hline D & NV & NV & NV & $\mathrm{NV}$ & $8-11$ & $10 \pm 0,95$ & $9-11$ & $10 \pm 0,45$ \\
\hline $\mathrm{A}$ & $\mathrm{NV}$ & $\mathrm{NV}$ & $\mathrm{NV}$ & $\mathrm{NV}$ & $9-12$ & $11 \pm 0,83$ & $10-12$ & $11 \pm 0,55$ \\
\hline
\end{tabular}

cabeça, enquanto na bexiga natatória e ao longo do intestino a pigmentação é interna. Ventralmente há uma concentração na superfície abdominal e, a partir de 3,50 mm, ocorre o aparecimento de cromatóforos internos, formando uma linha horizontal na altura do olho e, externamente sobre o corpo. Com 4,25 mm, estes são verificados ao redor da boca. O intestino é funcional e curto, a abertura anal não alcança a região mediana do corpo. O focinho é arredondado e a boca é terminal. As narinas são simples. Os olhos são bem pigmentados e esféricos e o opérculo encontra-se totalmente formado. A membrana embrionária ("finfold") é hialina e desprovida de pigmentação, circundando o corpo dorsal e ventralmente. Os botões das nadadeiras peitorais encontram-se presentes, porém não apresentam raios. A notocorda não se encontra flexionada e, é visível por transparência. A bexiga natatória encontra-se inflada. O número total de miômeros varia de 23 a 32 ( 12 a 17 pré e 10 a 15 pós-anal).

Estágio de flexão (Figura 2b): Os indivíduos apresentam comprimento padrão variando de 5,33 a 7,33 mm. O saco vitelino encontra-se totalmente absorvido. A pigmentação é semelhante ao estágio anterior, porém intensifica-se moderadamente e com $6,25 \mathrm{~mm}$, ocorre o aparecimento de cromatóforos na nadadeira dorsal, formando posteriormente, uma mácula. O intestino é longo e a abertura anal localiza-se na região posterior ao meio do corpo. A boca é pequena e a maxila alcança a órbita do olho e as membranas branquiais são livres do istmo e entre si. A membrana embrionária ainda está presente, entretanto observa-se a formação dos raios das nadadeiras caudal, anal e dorsal, os quais se iniciam com 6,08, 6,33 e 6,41 mm, respectivamente. Com aproximadamente $5,33 \mathrm{~mm}$, a notocorda encontra-se flexionada e, a partir de 5,75 mm um prolongamento em forma de "S" se diferencia em um dos raios da nadadeira caudal. O número total de miômeros varia de 29 a 32 (16 a 20 pré e 11 a 14 pós-anal).

Estágio de pós-flexão (Figura 2c-e): Os indivíduos possuem comprimento padrão variando de 7,40 a 13,28 mm. A pigmentação intensifica-se sobre todo o corpo, surgindo algumas faixas transversais e, em conseqüência da formação dos tecidos, os cromatóforos internos são menos visíveis. Entre os raios das nadadeiras anal e caudal, os cromatóforos estão presentes em pequeno número. $\mathrm{O}$ 
focinho torna-se pontiagudo e a partir de $12,86 \mathrm{~mm}$ a narina é dupla. O botão da nadadeira pélvica está presente no início deste estágio e, a partir de 8,25 mm, os raios já encontram-se formados. As nadadeiras são desprovidas de espinhos e, posteriormente, os raios se bifurcam. A seqüência de segmentação dos raios das nadadeiras é: caudal $(7,60 \mathrm{~mm})$, dorsal $(8,25 \mathrm{~mm})$, anal $(9,00 \mathrm{~mm})$ e pélvicas $(12,00 \mathrm{~mm})$. Em relação à nadadeira peitoral, apenas em 12,12 mm os raios começam a se formar. A membrana embrionária é encontrada apenas na região ventral do corpo, sendo totalmente absorvida com cerca de $9 \mathrm{~mm}$. A linha lateral é ausente e a bexiga natatória encontra-se inflada, não estando mais visível por transparência. As escamas são visíveis a partir de 7,75 mm. Neste estágio não é mais possível visualizar os miômeros.

Relações morfométricas: Neste período, o corpo varia de longo a moderado (12,50 a 27,48\%) e o comprimento da cabeça de pequeno a moderado (14,29 a 32,82\%). O olho varia de grande a moderado $(58,33$ a $29,28 \%)$. O comprimento do focinho $(10$ a $27,56 \%)$ e as distâncias pré-peitoral (12,2 a 34,35\%), pré-dorsal (56,58 a 63,48\%) e pré-anal $(69,17$ a $74,42 \%)$ apresentam aumento durante o desenvolvimento, enquanto a altura da cabeça diminui passando de $100 \%$ para 53,33\% (Tabela 1).

\section{Caracterização do período juvenil (Figura 2f)}

Neste período, os indivíduos possuem comprimento padrão variando de 13,14 a 31,00 mm. Apresentam uma faixa longitudinal de pigmentos entre o focinho e o opérculo, enquanto a cabeça e o corpo são bastante pigmentados, apresentando várias manchas irregulares. Os cromatóforos são verificados também entre os raios das nadadeiras e nas margens das nadadeiras dorsal e anal. Uma mácula evidente é observada na região mediana da nadadeira dorsal. Os indivíduos apresentam características semelhantes às de um adulto, como escamas e raios das nadadeiras formados e segmentados. A boca é pequena e terminal e a narina é dupla. O número total de raios é: P. 8-14, V. 7-9, D. 9-11 e A. 10-12.

Relações morfométricas: O comprimento (31,06 a 22,63\%) e a altura da cabeça $(77,72$ a $64,58 \%)$, o diâmetro do olho (42,15 a $23,95 \%)$, a altura do corpo (30,93 a 21,55\%) e as distâncias prépeitoral (30,75 a 17,11\%), pré-dorsal (64,22 a 50,83\%) e pré-pélvica $(56,29$ a $45,25 \%)$ apresentam diminuição ao longo do desenvolvimento, enquanto o comprimento do focinho $(16,59$ a $28,27 \%)$ e a distância pré-anal (64,17 a 75,73\%) aumentam (Tabela 1).

\section{Relações corporais}

Entre as variáveis morfométricas relacionadas, apenas duas apresentaram crescimento alométrico, o comprimento do focinho (alométrico positivo) e o diâmetro do olho (alométrico negativo), ambos em relação ao comprimento da cabeça (Tabela 2). O crescimento da altura da cabeça (AC/CC), comprimento da cabeça, altura do corpo e distância pré-peitoral (CC/CP, ACO/CP e FNP/CP) foram melhor representados pelo modelo de regressão linear tipo piecewise, ou seja, houve um crescimento inicial semelhante à variável independente $\mathrm{e}$ uma mudança abrupta, posteriormente ao ponto de quebra. As distâncias pré-pélvica, pré-dorsal e pré-anal apresentaram crescimento isométrico (Tabela 2).

\section{Discussão}

A grande concentração de pigmentos apresentada por $P$. australis durante o seu desenvolvimento inicial é comum em peixes que têm como habitat preferencial áreas litorâneas cobertas por vegetação aquática (Machado-Allison 1987). Mudanças no padrão de pigmentação ocorrem quando as larvas passam a explorar as zonas litorâneas das áreas inundadas, intensamente cobertas por macrófitas aquáticas, tais como desenvolvimento de bandas, máculas e outras marcas no corpo e região da cabeça (Hoplias aff. malabaricus (Bloch, 1794), Hoplosternum littorale (Hancock, 1828), Leporinus spp., Prochilodus lineatus (Valenciennes, 1836), Schizodon spp., Serrasalmus spp.) (Nakatani et al. 2004). Essa intensa pigmentação provavelmente está associada à camuflagem para evitar a predação (Nakatani et al. 1997). Em relação às larvas de Pyrrhulina filamentosa Valenciennes, 1847 e Copella carsevennensis (Regan, 1912) (Ponton \& Mérigoux 2001), P. australis possui pigmentação semelhante, porém, com maior concentração de cromatóforos no dorso, tanto na cabeça quanto ao longo do corpo, e maior evidência da mácula na nadadeira dorsal.

Outro aspecto comum às espécies de Lebiasinidae é o prolongamento da notocorda em forma de filamento. Esta estrutura assemelhase a um apêndice, que ao término do período larval é incorporado aos raios da nadadeira caudal. Esta característica é extremamente importante na identificação de larvas de $P$. australis, uma vez que é exclusiva desta espécie, diferenciando-a de todas as demais encontradas na planície alagável do Alto Rio Paraná (Nakatani et al. 2001).

Algumas espécies desta família, tais como Copeina arnoldi (Regan, 1912), P. filamentosa, Lebiasina (=Piabucina) pleurotaenia

Table 2. Linear, quadratic and piecewise linear regression statistics for morfometric variables in larvae and juveniles of Pyrrhulina australis. $\mathrm{R}^{2}=$ coefficient of determination, $\mathrm{L}=$ linear regression, $\mathrm{Q}=$ quadratic regression, $\mathrm{S}=$ piecewise linear regression, $\mathrm{MM}=\mathrm{best}$ model, $\mathrm{PQ}=\mathrm{breakpoint}$, a and $\mathrm{b}=$ regression parameters. Values in bold showed $\mathrm{p}<0.05$.

Tabela 2. Estatísticas das regressões linear, quadrática e piecewise para as variáveis morfométricas obtidas em relação ao comprimento da cabeça e padrão em larvas e juvenis de Pyrrhulina australis. $\mathrm{R}^{2}=$ coeficiente de determinação, $\mathrm{L}=$ regressão linear, $\mathrm{Q}=$ regressão quadrática, $\mathrm{S}=$ regressão "piecewise", $\mathrm{MM}=$ melhor modelo, $\mathrm{PQ}=$ ponto de quebra, a e $\mathrm{b}=$ parâmetros das regressões e $\mathrm{n}=$ número de indivíduos analisados. Valores em negrito apresentaram $\mathrm{p}<0,05$.

\begin{tabular}{|c|c|c|c|c|c|c|c|c|c|c|c|c|c|}
\hline \multirow[t]{2}{*}{ Relação } & \multicolumn{3}{|c|}{$\mathbf{R}^{2}$} & \multicolumn{3}{|c|}{ F-teste } & \multirow[t]{2}{*}{ MM } & \multirow[t]{2}{*}{$\mathbf{P Q}$} & \multirow[t]{2}{*}{ a1 } & \multirow[t]{2}{*}{ b1 } & \multirow[t]{2}{*}{ a2 } & \multirow[t]{2}{*}{ b2 } & \multirow[t]{2}{*}{ n } \\
\hline & $\mathbf{L}$ & $\mathbf{Q}$ & $\mathbf{S}$ & $Q / L$ & S/Q & $\mathrm{S} / \mathrm{L}$ & & & & & & & \\
\hline $\mathrm{CF} / \mathrm{CC}$ & 0,96 & 0,97 & 0,98 & 54,02 & $-1,24$ & 25,60 & $Q$ & - & 0,02 & 0,16 & - & - & 80 \\
\hline DOL/CC & 0,96 & 0,97 & 0,97 & 16,93 & $-1,41$ & 7,49 & $Q$ & - & $-0,01$ & 0,44 & - & - & 80 \\
\hline $\mathrm{AC} / \mathrm{CC}$ & 0,99 & 0,99 & 0,99 & 4,86 & 15,40 & 10,59 & $S$ & 1,82 & 0,65 & 0,07 & 0,65 & 0,32 & 80 \\
\hline $\mathrm{CC} / \mathrm{CP}$ & 0,97 & 0,97 & 0,99 & 7,22 & 104,96 & 61,03 & $S$ & 2,55 & 0,36 & $-0,63$ & 0,22 & 0,97 & 80 \\
\hline $\mathrm{ACO} / \mathrm{CP}$ & 0,96 & 0,96 & 0,97 & $-14,99$ & 53,36 & 14,02 & $S$ & 2,26 & 0,34 & $-0,75$ & 0,23 & 0,43 & 80 \\
\hline $\mathrm{FNP} / \mathrm{CP}$ & 0,94 & 0,95 & 0,97 & 15,45 & 52,94 & 39,48 & $S$ & 2,61 & 0,37 & $-0,67$ & 0,20 & 1,20 & 80 \\
\hline FNL/CP & 0,99 & 0,99 & 0,99 & 6,76 & 6,95 & 7,41 & $L$ & - & 0,48 & 0,46 & - & - & 40 \\
\hline FND/CP & 0,98 & 0,98 & 0,98 & 1,09 & 2,12 & 1,62 & $L$ & - & 0,61 & 0,041 & - & - & 40 \\
\hline FNA/CP & 0,98 & 0,98 & 0,98 & $-0,88$ & 1,88 & 0,49 & $L$ & - & 0,75 & $-0,36$ & - & - & 40 \\
\hline
\end{tabular}


Regan, 1903 e Copella sp., são pertencentes à guilda dos guardadores (aerófilos) (Balon 1975), ou seja, depositam seus ovos embaixo de folhas largas ou rochas que se projetam da água, os quais são cuidados pelo macho após a fecundação (Machado-Allison 1987, Vazzoler 1996, Helfman et al. 1997). Outras, como Pyrrhulina brevis Steindachner, 1876 e Pyrrhulina laeta (Cope, 1872) não são guardadoras e depositam seus ovos em cavidades (Baensch \& Riehl 1985). Apesar de não sabermos ao certo a que guilda reprodutiva P. australis pertence, a absorção tardia do saco vitelino (estágio de flexão) sugere a possibilidade de ser uma espécie guardadora, visto que nesta guilda, durante a transição da alimentação endógena para a exógena, as espécies apresentam tamanho relativamente grande (Balon 1984). Além disso, dificilmente indivíduos menos desenvolvidos são encontrados no ambiente, o que pode indicar algum grau de cuidado e também explicar a ausência de larvas em estágios iniciais de desenvolvimento (larval vitelino) neste estudo.

O surgimento de novas estruturas ao longo do desenvolvimento é importante, pois reflete uma ampla escala de adaptações que influenciam a sobrevivência no estágio larval. Em $P$. australis, a permanência da membrana embrionária nos estágios mais avançados, pode influenciar positivamente a taxa de sobrevivência e o sucesso no ambiente. Embora a explicação para a função desta estrutura ainda seja especulativa, existem, segundo Hunter (1972) e Van Snik et al. (1997), três hipóteses que tentam explicar a sua importância: utilização na locomoção e orientação das larvas com baixo valor de Reynolds (capacidade do organismo de se movimentar em um ambiente viscoso); participação na respiração; e importância na captura de presas, através da propulsão do corpo.

De acordo com Strauss (1984), o tamanho do olho está diretamente relacionado com o tipo de presa consumida. Segundo Abelha et al. (2001) dietas distintas dentro de uma mesma espécie são freqüentemente encontradas conforme os estágios de desenvolvimento dos indivíduos, decorrentes das diferenças na demanda energética e nas limitações morfológicas. No caso de $P$. australis, quando adulto, a alimentação se caracteriza por ser, principalmente, invertívora (Bulla et al. 2005), entretanto, a mudança no tamanho do focinho e no diâmetro do olho possivelmente implicam em dietas diferenciadas durante o desenvolvimento. Situação o qual pode ser observada em C. carsevennensis e $P$. filamentosa, as quais se alimentam de larvas de inseto e microcrustáceos nos estágio iniciais de desenvolvimento passando a incluir indivíduos maiores em sua dieta no período juvenil (Ponton \& Mérigoux 2001).

As relações entre as variáveis que apresentaram crescimento abrupto, ou seja, com pontos de quebra, podem estar associadas ao limiar entre os períodos de desenvolvimento, como por exemplo, o larval e o juvenil ou a etapas dentro de um mesmo período (por exemplo, pré-flexão para flexão). Segundo Kováč et al. (1999), este ponto de quebra é considerado significativo se for associado a algum evento morfológico, fisiológico e/ou de sobrevivência. Neste caso, a altura e o comprimento da cabeça, a altura do corpo e a distância pré-peitoral, apresentaram interrupção do crescimento dentro do estágio de pós-flexão, sugerindo que neste intervalo ocorre a maior parte da remodelação da forma externa do corpo.

O crescimento da cabeça provavelmente está relacionado ao desenvolvimento dos aparatos branquiais e ao início da alimentação exógena (Blaxter 1988), enquanto a mudança do corpo de longo para moderado (relação $\mathrm{ACO} / \mathrm{CP}$ ) é conseqüência do desenvolvimento da musculatura e do trato digestório. Assim, este padrão pode estar vinculado a uma maior exigência por parte do ambiente, em relação à competição e captura de alimento, acelerando assim, o desenvolvimento corporal.

Apesar das variáveis distâncias pré-pélvica, pré-dorsal e pré-anal terem apresentado como melhor modelo de crescimento a regressão linear, existe a possibilidade de estes resultados terem sofrido interferência do pequeno número de indivíduos analisados, já que estas estruturas surgem apenas no final do período larval.

Com base nestes resultados, podemos concluir que o padrão de pigmentação e o prolongamento da notocorda em forma de filamento são importantes características que permitem diferenciar $P$. australis de outras espécies na planície alagável do Alto Rio Paraná. As principais mudanças na morfometria (metamorfose) ocorrem principalmente no final do período larval e no começo do juvenil. É possível que estas mudanças estejam associadas a alterações comportamentais da espécie, como por exemplo, obtenção de alimento e exploração do hábitat; no entanto esta observação precisa ser ainda investigada.

\section{Agradecimentos}

Agradecemos ao Núcleo de Pesquisas em Limnologia, Ictiologia e Aqüicultura (Nupélia), pelo suporte logístico; aos programas PADCT/ CIAMB e PIE/PELD/CNPq, bem como à ELETROBRÁS, pelo apoio financeiro; e aos amigos Alfredo Soares, Francisco Alves Teixeira, Sebastião Rodrigues, Valdir Aparecido Capatti e Valmir Alves Teixeira, pelo auxílio nas coletas de campo. Os autores Andréa Bialetzki, Gilmar Baumgartner e Maristela Cavicchioli Makrakis agradecem ao CNPq, pela concessão de bolsa de produtividade de pesquisa.

\section{Referências}

ABELHA, M.C.F., AGOSTINHO, A.A. \& GOULART, E. 2001. Plasticidade trófica em peixes de água doce. Acta Scientiarum 23(2):425-434.

AHLSTROM, E.A. \& BALL, O.P. 1954. Description of egg and larvae of jack mackarel (Trachurus symetricus) and distribution and abundance of larvae in 1950 and 1951. Fish. Bull. 56: 209-245.

AHLSTROM, E.H. \& MOSER, H.G. 1976. Eggs and larvae of fishes and their role in systematic investigations and in fisheries. Rev. Trav. Inst. Pech. Marit. 40(3):285-402.

ALHSTROM, E.H., BUTLER, J.L. \& SUMIDA, B.Y. 1976. Pelagic stromateoid fishes (Pisces, Perciformes) of the Eastern Pacific: kinds, distributions, and early life histories and observations of five of these from the Northwest Atlantic. Bull. Mar. Sci. 26(3):285-402.

BAENSCH, H.A. \& RIEHL, R. 1985. Aquarien atlas. Band 2. Mergus, Verlag für Naturund Heimtierkunde GmbH, Melle, 1216 p.

BALON, E.K. 1975. Reproductive guilds of fishes: a proposal and definition. J. Fish. Res. Board Can. 32(6):821-864.

BALON, E.K. 1984. Reflections on some decisive events in the early life of fishes. Trans. Am. Fish. Soc. 113(2):178-185.

BIALETZKI, A., SANCHES, P.V., BAUMGARTNER, G. \& NAKATANI, K. 1998. Caracterização morfológica e distribuição temporal de larvas e juvenis de Apareiodon affinis (Steindachner) (Osteichthyes, Parodontidae) no alto rio Paraná, Paraná. Rev. Bras. Zool. 15(4):1037-1047.

BLAXTER, J.H.S. 1988. Pattern and variety in development. In Fish Physiology (W.S. Hoar \& D.J. Randall, eds). Academic Press, London. p.1-58.

BUCKUP, P.A. 1999. Sistemática e biogeografia de peixes de riachos. In Ecologia de peixes de riachos. (E.P. Caramaschi, R. Mazzoni \& P.R. Peres-Neto, eds). Série Oecologia Brasiliensis, vol. VI. PPGE-UFRJ, Rio de Janeiro, p. 91-138.

BULLA, C.K., GOMES, L.C. \& AGOSTINHO, A.A. 2005. Ictiofauna. Fauna associada a bancos flutuantes de macrófitas. In A Planície alagável do rio Paraná (A.A Agostinho, S.M. Thomaz, L. Rodrigues \& L.C. Gomes, coord.). UEM, Maringá, p. 126-131. (Relatório técnico) http://www. peld.uem.br/Relat2005/pdf/12_Ictiofauna1_2005.pdf (último acesso em: 12/06/2007)

GRAÇA, W.J. \& PAVANELLI, C.S. 2007. Peixes da planície de inundação do alto rio Paraná e áreas adjacentes. EDUEM, Maringá, 241 p.

FUIMAN, L.A. 1983. Growth gradients in fish larvae. J. Fish Biol. 23:117-123 
HELFMAN, G.S., COLLETE, B.B. \& FACEY, D.E. 1997. The diversity of fishes. Blackwell Science Editorial Offices, Massachusetts, 528 p.

HUNTER, J.R. 1972. Swimming and feeding behavior of larval anchovy Engraulis mordax. Fish. Bull. 70(3):821-838.

KOVÁČ, V., COPP, G.H. \& FRANCIS, M.P. 1999. Morphometry of the stone loach, Barbatula barbatula: do mensural characters reflect the species life history thresholds? Environ. Biol. Fish. 56(1-2):105-115.

LEIS, J.M. \& TRNSKI, T. 1989. The larvae of Indo-Pacific shorefishes. University of Hawaii Press, Honolulu, $371 \mathrm{p}$.

MACHADO-ALLISON, A. 1987. Los peces de los llanos de Venezuela: un ensayo sobre su historia natural. Universidad Central de Venezuela, Caracas, $144 \mathrm{p}$.

NAKATANI, K., BAUMGARTNER, G. \& CAVICCHIOLI, M. 1997. Ecologia de ovos e larvas de peixes. In A planície de inundação do Alto Rio Paraná: aspectos físicos, biológicos e econômicos. (A.E.A.M. Vazzoler, A.A. Agostinho \& N.S. Hahn, eds). EDUEM, Maringá, p.281-306.

NAKATANI, K., AGOSTINHO, A.A., BAUMGARTNER, G., BIALETZKI, A., SANCHES, P.V., MAKRAKIS, M.C. \& PAVANELLI, C.S. 2001. Ovos e larvas de peixes de água doce: desenvolvimento e manual de identificação. EDUEM, Maringá, 378 p.

NAKATANI, K., BIALETZKI, A. \& SANCHES, P.V. 2004. Eggs and larvae of fishes in the Upper Paraná River floodplain. In Struture and functioning of the Paraná River and its floodplain (A.A. Agostinho, L. Rodrigues, L.C. Gomes, S.M. Thomaz \& L.E., Miranda, eds). EDUEM, Maringá, p.157-161.
PONTON, D. \& MÉRIGOUX, S. 2001. Description and ecology of some early life stages of fishes in the river Sinnamary (French Guiana, South America). Folia Zool. 50(1):1-116.

SNYDER, D.E. 1981. Contributions to a guide to the cypriniform fish larvae of the Upper Colorado River System in Colorado. United States, Bureau of Land Management, Colorado Office, Denver. (Biological Sciences Series 3).

SOKAL, R.R. \& ROHLF, F.J. 1981. Biometry. The principle and practice of statistics in biological research. W. H. Freeman, San Francisco, 859 p.

STRAUSS, R.E. 1984. Allometry and functional feeding morphology in haplochromine cichlids. In Evolution of fish species flocks (A.A. Echelle \& I. Kornfield, eds). University of Maine Press, Orono, p.217-229.

VAN SNIK, G.M.J., VAN DEN BOOGAART, J.G.M. \& OSSE, J.W.M. 1997. Larval growth patterns in Cyprinus carpio and Clarias gariepinus with attention to the finfold. J. Fish Biol. 50(6):1339-1352.

VAZZOLER, A.E.M. 1996. Biologia da reprodução de peixes teleósteos: teoria e prática. EDUEM, Maringá, 169 p.

WEITZMAN, M. \& WEITZMAN, S.H. 2003. Family Lebiasinidae (Pencil fishes). In Check list of the freshwater fishes of South and Central America (R.E. Reis, S.O. Kullander \& C.J. Ferraris Jr., eds). Edipucrs, Porto Alegre, p.241-250.

Recebido em 27/05/09

Versão reformulada recebida em 26/08/09

Publicado em 05/11/09 